\title{
BER Evaluation of Rayleigh Fading Channel with M-PSK Modulation
}

\author{
Rakesh Kumar \\ PG Scholar \\ NIIST, Bhopal
}

\author{
Akhilesh Jain \\ Research Guide \\ NIIST, Bhopal
}

\begin{abstract}
Modern wireless communication system is getting better for the new generation of data communication technology, because it have to facilitates the user to communicate and share information through various wirelessly connected devices in moving. The research works are delicately exploring new dimensions of the technology and fixing the bugs day by day. The every researchers aim to explore new techniques and analyze the existing technologies to make technology easier for the subscribers having several features. In the same context this work also analyzing for estimation of channel with utilizing pilot assisted scheme and spatial diversity using different number of antennas at the transmitter and receiver side to make system more efficient for random channel behavior. The methodology of this work having better error probability than the existing work done on the same context. The proposed system utilizes multi antenna diversity for $4 \times \mathrm{xM}$ and $2 \mathrm{xM}$,configurationswhere $\mathrm{M}$ is number of receiver antennas and modulation scheme is 32-PSK.
\end{abstract}

\section{Keywords}

Pilot Assisted, Spatial Diversity, 32-PSK, MIMO.

\section{INTRODUCTION}

Recently, a worldwide convergence has occurred for the use of Orthogonal Division Frequency Multiplexing as an emerging technology for high data rates. In particular, the wireless local network systems such as WiMax, WiBro, WiFi etc., and the emerging fourth-generation (or the so-called 3.9G) mobile systems are all OFDM based systems. OFDM is a digital multi-carrier modulation conspires, which utilizes countless spaced orthogonal sub-carriers that is especially reasonable for frequency-selective channels and high information rates [1], and [2]. This system changes a frequency-specific wide-band channel into a gathering of nonparticular thin band channels, which makes its vigorous against large defer spreads by safeguarding orthogonality in the frequency area. Additionally, the introduction of an asserted cyclic prefix at the transmitter decreases the multifaceted design at receiver to FFT get ready and one tap scalar equalizer at the receive.

OFDM is a multi-carrier modulation plot that encodes information onto a Radio Frequency (RF) signal. Not at all like regular single carrier modulation plans, for example, $\mathrm{AM} / \mathrm{FM}$ (amplitude or frequency modulation) that send just a single signal at any given moment utilizing one radio frequency, OFDM sends various rapid signals simultaneously on uncommonly figured, orthogonal carrier frequencies. The outcome is significantly more effective utilization of bandwidth and strong communications amid commotion and different interferences.
OFDM is modulation system known for its ability to direct multipath. In OFDM the quick data stream is isolated into $\mathrm{R}$ narrowband data streams, $\mathrm{R}$ identifying with the subcarriers or subchannels i.e. one OFDM image comprises of $\mathrm{R}$ images regulated by Quadrature Amplitude Modulation (QAM) or Phase Shift Keying (PSK). Thus the image term is R times longer than in a solitary carrier framework with a similar image rate. The image term is made considerably longer by adding a cyclic prefix to every image. For whatever length of time that the cyclic prefix is longer than the channel defer spread, OFDM offers Inter Symbol Interference (ISI) free transmission

The simplified equalization at receiver, however, requires knowledge of the channel over which the signal is transmitted. To encourage the estimation of the direct in an OFDM framework, (for example, WiMax, WiBro, WiFi, and 3.9/4G), known signals or pilots could be embedded in the transmitted OFDM image. Distinctive strategies can then be connected to appraise the channel utilizing these known pilots. The focus of this report is to investigate performance of different channel estimators for an OFDM-based 3.9G system. The outcome of the report is to recommend a channel estimation method for implementation and future study.

\subsection{Propagation Aspects}

In wire-line communication, the data transmission is primarily corrupted by statistically independent Gaussian noise, as known as the classical additive white Gaussian noise (AWGN). In absence of interference, the primary source of performance degradation in such wire-line channels is thermal noise generated at the receiver. Reliable communication in wireless or radio channels, however, becomes a difficult task as the transmitted data is not only corrupted by AWGN, but also suffers from inter-symbol interference (ISI), in addition to (large-scale and small-scale) fading as well as interference from other users.

\subsubsection{Small-scale Fading}

In a typical wireless communications system, the transmitted signal typically undergoes refractions, shadowing and various reflections due to the presence of various objects (buildings, trees, etc.) in the channel [5]. As a consequence the waves emitted by the receiver arrive at the receiver antenna over multiple paths, a phenomenon known as multipath propagation. The complete set of propagation paths between transmitter and receiver forms the multipath channel. Each path can be characterized by three parameters: delay, attenuation and phase shift.

In wireless mobile communications, surrounding objects, such as houses, buildings, trees and mountains provoke reflection, diffraction, scattering and shadowing of the transmitted signals and causes multipath propagation. This multipath effect causes the transmitted signals to arrive at the receiver 
with different phase angles, different amplitude and at different time intervals. The total of the received signals is consolidated in a ruinous or useful approach to recuperate the transmitted signal. The sufficiency vacillation of the received signal is called signal fading and is brought on by the frequency particular or time variation attributes of the multipath channel.

\subsubsection{Frequency Non Selective Channel}

The transmitted wireless signal is subject to multipath time delay spread or Doppler spread. Multipath channels with time delay spread can be divided into two categories: frequency non selective channel or frequency selective channel.

Under time dispersion, received signals undergo frequency non selective channels also known as flat fading, when the channel bandwidth is much larger than the bandwidth of the transmitted signal. Since the bandwidth of the applied signal is narrower than the channel bandwidth, flat fading channels are often referred to as narrowband channels.

\subsection{System Model}

MIMO technology constitutes a leap forward in wireless communication framework design. The technology offers various advantages that help meet the difficulties postured by both the hindrances in the wireless channel and additionally asset limitations. Notwithstanding the time and frequency measurements that are dimensions in regular single antenna wireless frameworks, the influences of MIMO are acknowledged by abusing the spatial measurement (gave by the various antennas at the transmitter and the receiver). The benefits of MIMO technology helps to achieve significant performance gains such as array gain, spatial diversity gain, spatial multiplexing gain and interference reduction.

\subsubsection{MIMO-OFDM Channel Estimation}

High data rate and strong reliability in wireless communication systems are becoming the dominant factors for a successful exploitation of commercial networks. MIMOOFDM, a new wireless broadband technology, has gained great popularity for its capability of high rate transmission and its robustness against multipath fading and other channel impairments. In an OFDM framework, the transmitter modulates the message bit stream into PSK/QAM images, performs IFFT on the images to change over them into time area signals, and sends them out through a wireless channel. The received signal is generally contorted by the channel qualities. In order to recover the transmitted bits, the channel effect must be estimated and compensated in the receiver.

\subsubsection{Pilot symbol based channel estimation}

The major challenge faced in MIMO-OFDM systems is to obtain the channel state information accurately and promptly for coherent detection of information symbols. The channel state data can be acquired through preparing based, blind and semi blind channel estimation. In training based channel estimation calculations, preparing images or pilot tones that are known from the earlier to the receiver, are multiplexed alongside the information stream for channel estimation. The blind channel estimation is carried out by evaluating the statistical information of the channel and certain properties of the transmitted signals.

\subsubsection{Least square (LS) channel estimation}

Training symbols can be used for channel estimation, usually providing a good performance. However, their transmission efficiencies are reduced due to the required overhead of training symbols such as preamble or pilot tones that are transmitted in addition to data symbols. The Least Square (LS) and Minimum Mean Square Error (MMSE) techniques are widely used for channel estimation when training symbols are available. The LS channel estimation method finds the channel estimate in such a way that the difference between the actual value and true value of channel parameters is minimized. Due to its simplicity, however, the LS method has been widely used for channel estimation. The pilot symbol based LS channel estimation method provides better performance of estimating the channel information accurately.

\subsubsection{Minimum mean square error (MMSE)}

The MMSE estimator utilizes the second request insights of the channel conditions to limit the mean square mistake. The MMSE estimator yields much preferable execution over LS estimators, particularly under the low SNR situations. A noteworthy disadvantage of the MMSE estimator is its high computational multifaceted nature, particularly if grid reversals are required each time the information in changes.

\section{PROPOSED METHODOLOGY}

The proposed multiple input multiple output (MIMO )system is based on the M-psk modulation scheme having antenna diversity of $4 \mathrm{xM}$ and $2 \mathrm{xM}$ configurations.

\subsection{M-Phase Shift Keying (M-PSK)}

PSK relies on carrier changing between distinct phases of the signal to define the status of information being transmitted. PSK default is considered for binary (two) levels of phase modulations. PSK is considered a very efficient process of data delivery because of low bit error rates in the delivery. A number of variations of PSK are used in wireless networking systems, among which are BPSK, QPSK. Most of current wireless systems employ some form of PSK.M-PSK regulates an info signal utilizing $\mathrm{M}$-ary phase shift keying (PSK) and returns a complex baseband yield. The modulation arranges, $\mathrm{M}$, which is equal to the quantity of focuses in the signal group of stars, is dictated by the M-ary number parameter.

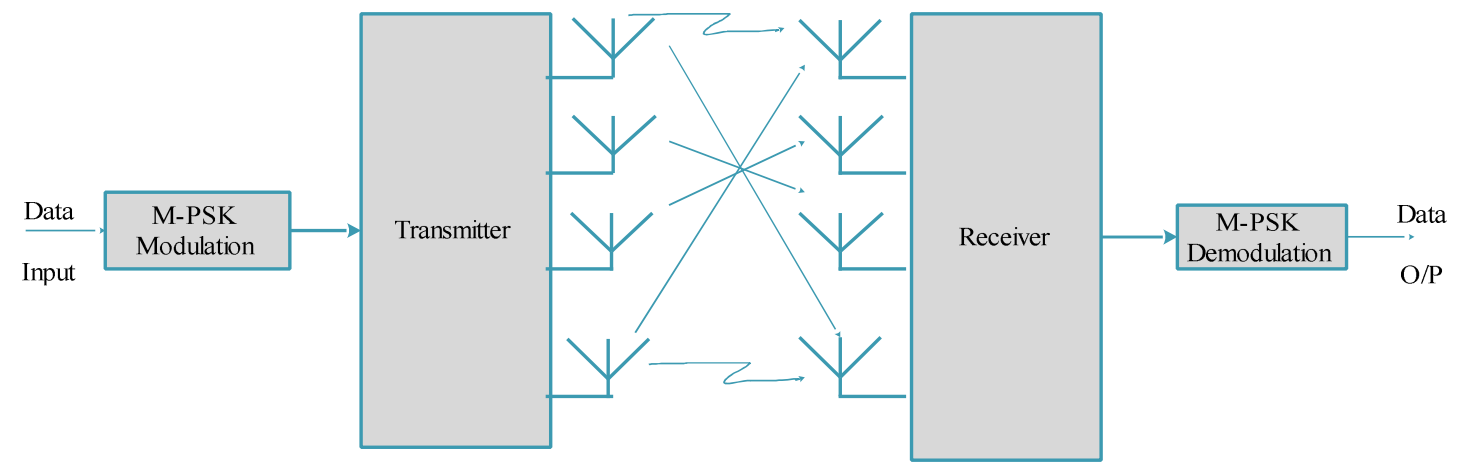

Fig 1:Block Diagram of Proposed work. 
The methodology of this work having better error probability than the existing work done on the same context. Figure 3.1 illustrate the block diagram of proposed MIMO system. $\mathrm{m}$ number of antennas at both transmitter and receiver end. in the proposed work we are using M-PSK modulation scheme. Multi-carrier modulations is used for MIMO system that utilization orthogonal waveform for modulate the sub-carriers are called orthogonal frequency division multiplex (OFDM) schemes. Since the sub-carriers are modulated by orthogonal waveforms, the sub-carriers are allowed to have overlapping spectrum, in this way accomplishing higher spectrum efficiency.

\subsection{Flow of Process}

The flow of process has illustrated in figure 3.2 the flow of process the process has start with the initialization of parameters and generate random signal which is to be transmitted for the experimental purpose .modulate generated random signal with M-array phase shift keying modulation (M-PSK). Initialize MIMO channel Model as demonstrated in figure 3.1 and start symbol transmission using pilots. add noise to transmission signal for experimental purpose . Demodulate received signal with $\mathrm{M}$ - psk demodulation .calculate bit error rate and mean of the received signal.finally display result on screen.
Start

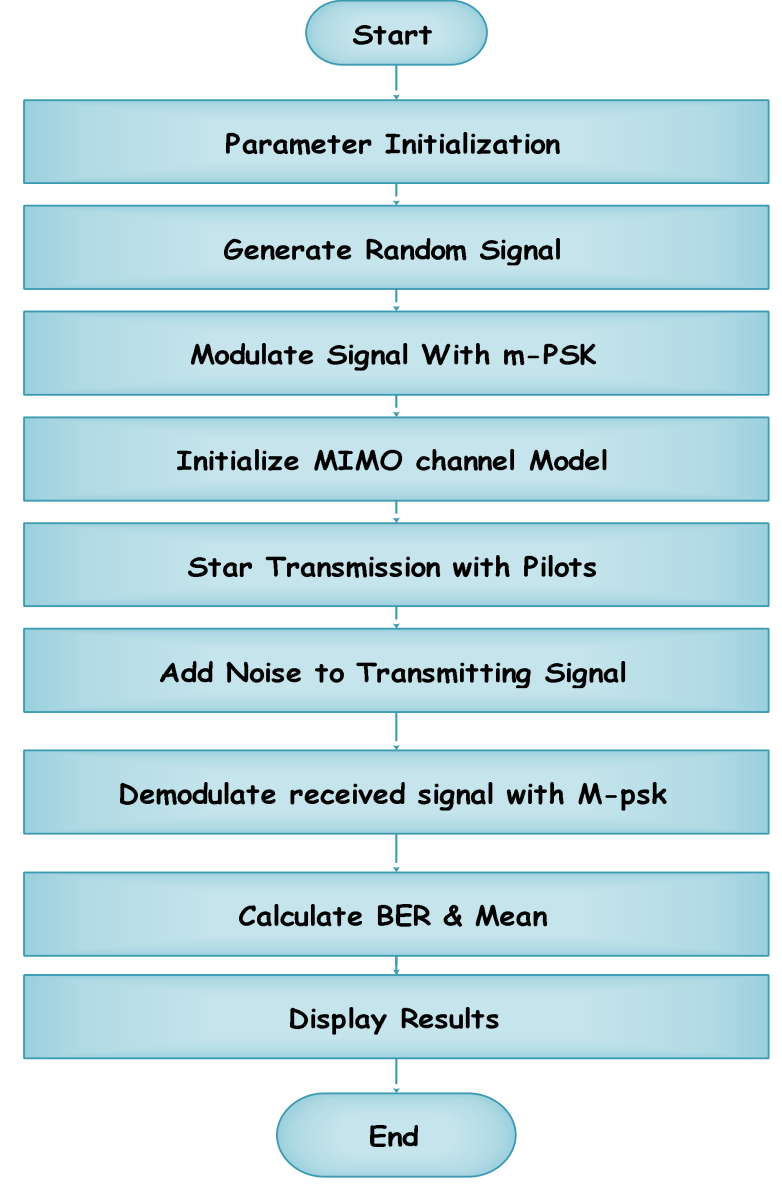

Figure 2: Process flow of proposed system.

\section{SIMULATION RESULTS}

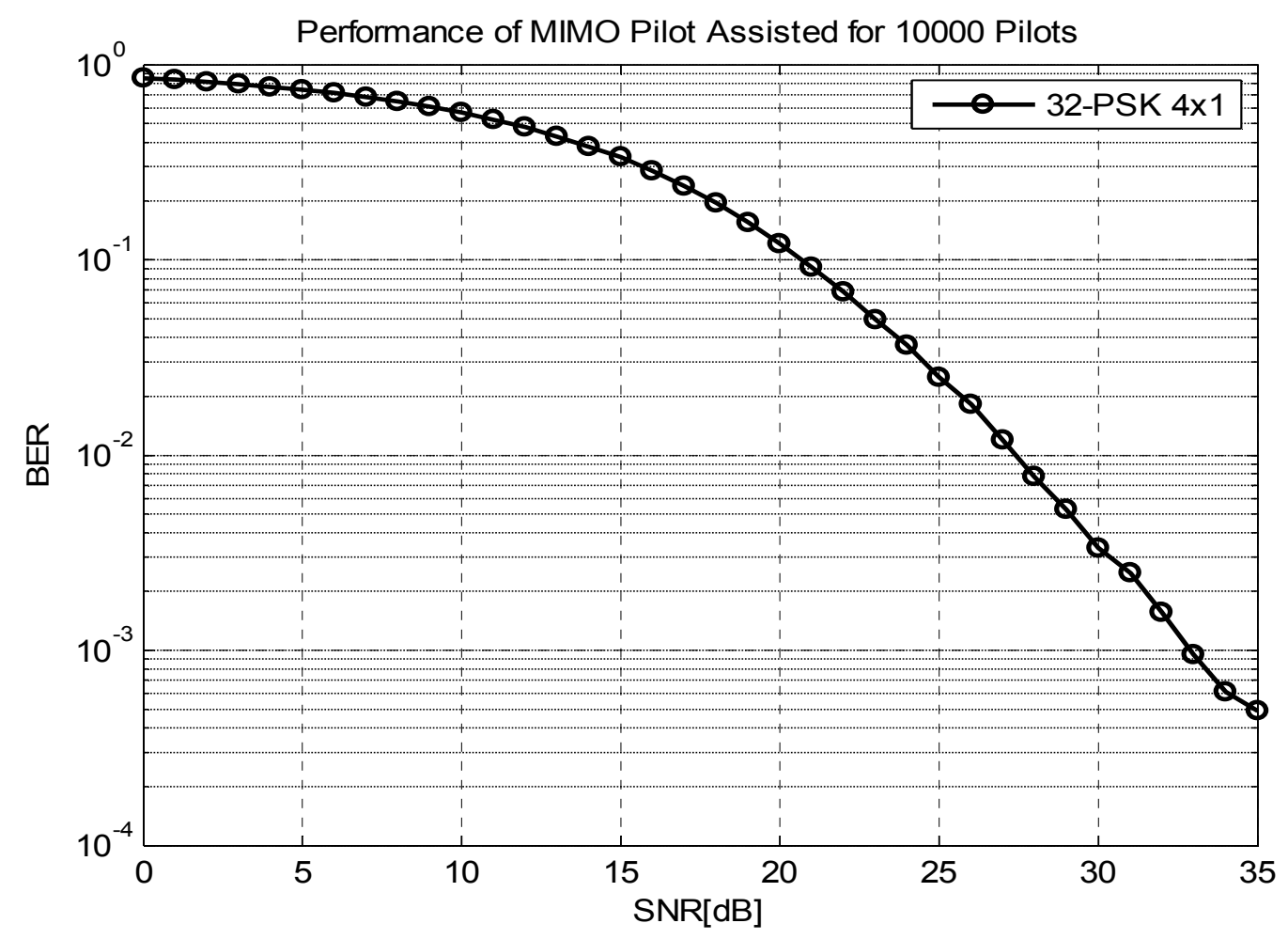

Fig.3: BER Performance of Proposed System with 10K Pilots and 4 transmitter and 1 receiver antenna 


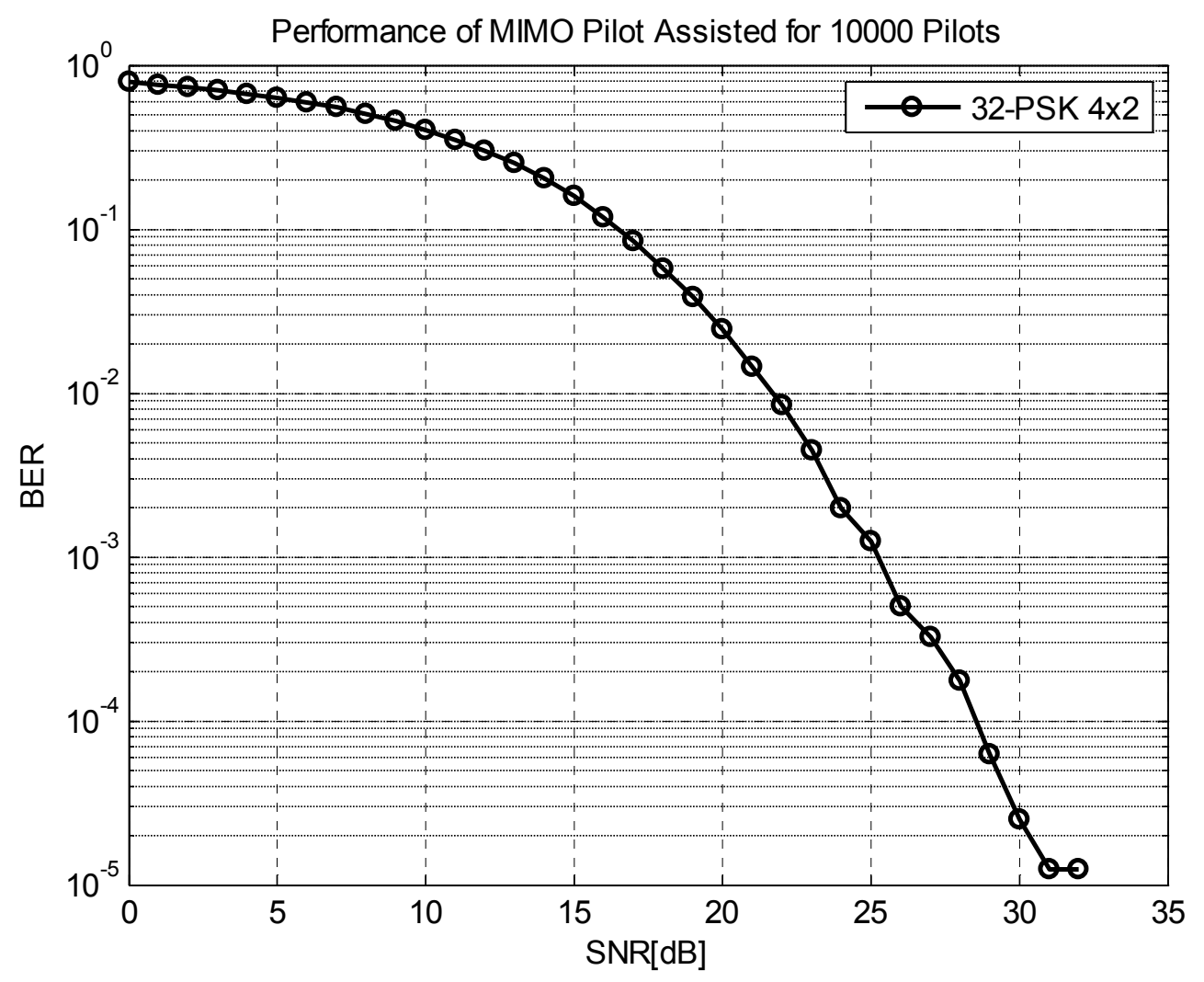

Fig. 4:BER Performance of Proposed System with 10K Pilots and 4 transmitter and 2 receiver antenna

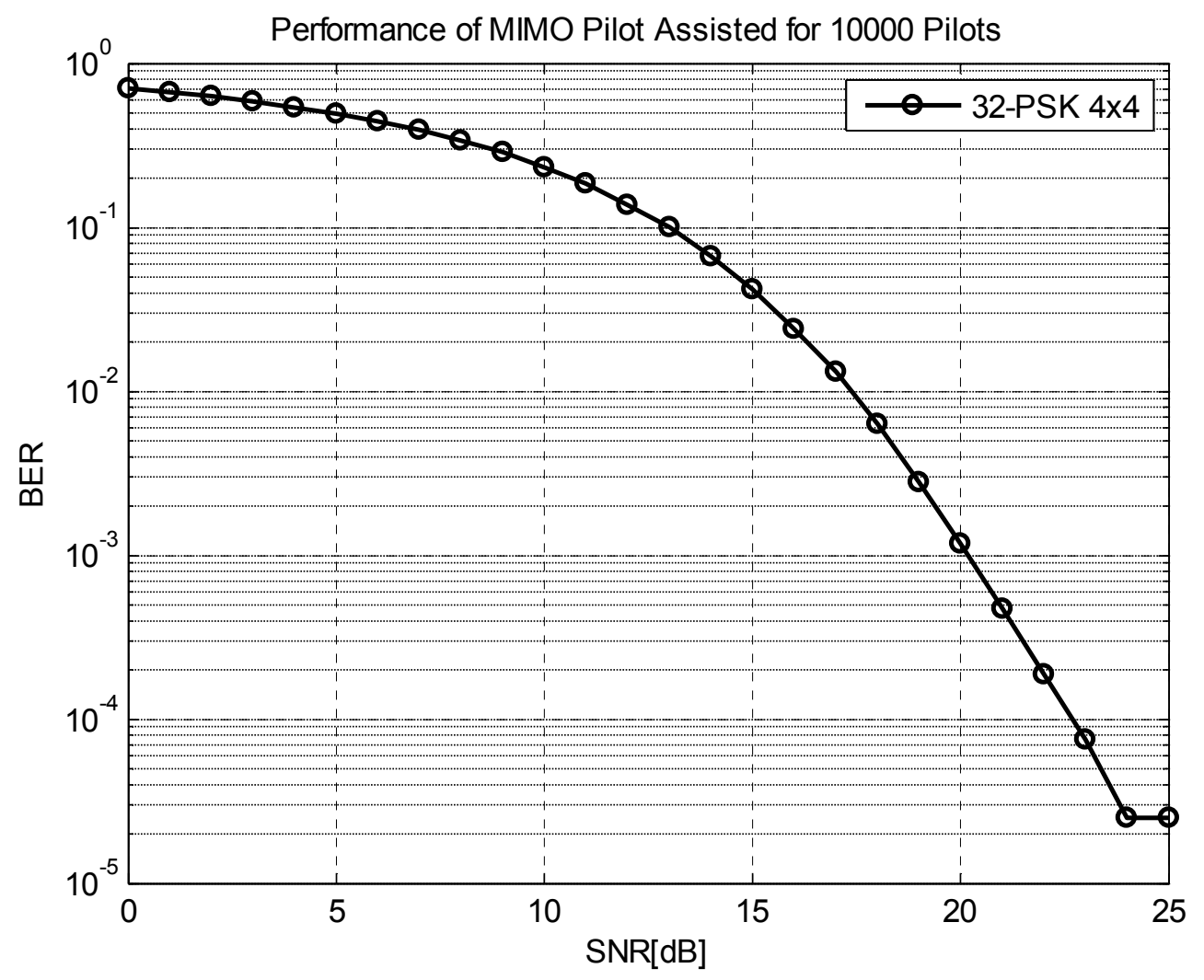

Fig.5: BER Performance of Proposed System with 10K Pilots and 4 transmitters and 4 receiver antenna 


\section{CONCLUSION}

The results of the proposed model after simulation is displayed in the previous section and the analysis of the system with BER and spatial diversity architecture used conclude that the proposed approach is better with the reduced error probability in the system. The $2 \mathrm{xM}$ and $4 \mathrm{xM}$ configuration giving better BER for higher signal power range keeping number of receivers (M) lower or equal to number of transmitters. But when number of receivers is increased than the transmitters BER for all the signal powers perform better than the existing work which was pilot assisted STBC MISO system.

The MIMO architecture shown that the performance can be better than MISO used with 32-PSK modulation, and it can be more better with efficient modulation technique like QAM instead of PSK, and the digital filtering or detections techniques will make proposed system better.

\section{REFERENCES}

[1] M. Raju and K. A. Reddy, "Evaluation of BER for AWGN, Rayleigh fading channels under M-QAM modulation scheme," 2016 International Conference on Electrical, Electronics, and Optimization Techniques (ICEEOT), Chennai, 2016, pp. 3081-3086.

[2] J. A. Sheikh, Uzma, S. A. Parah and G. M. Bhat, "Bit Error Rate (BER) improvement of Multiple Input Multiple Output Orthogonal Frequency Division Multiplexing (MIMO-OFDM) system using bit level scrambling," 2015 Annual IEEE India Conference (INDICON), New Delhi, 2015, pp. 1-6.

[3] R. Yoshizawa and H. Ochiai, "Mutual Information and Coded BER Analysis of PAPR Reduced OFDM System with Active Constellation Extension," 2015 IEEE Global Communications Conference (GLOBECOM), San Diego, CA, 2015, pp. 1-6.

[4] Y. Goto et al., "BER characteristic of optical-OFDM using OCI," 2014 IEEE Asia Pacific Conference on Circuits and Systems (APCCAS), Ishigaki, 2014, pp. 328-331.

[5] M. Thanigasalam and P. Dananjayan, "BER analysis of OFDM receiver using MMSE channel estimation and modified PTS combined with interleaving," 2014 IEEE International Conference on Advanced Communications, Control and Computing Technologies, Ramanathapuram, 2014, pp. 662-666.
[6] S. R. Chaudhary and M. P. Thombre, "BER performance analysis of MIMO-OFDM system using different equalization techniques," 2014 IEEE International Conference on Advanced Communications, Control and Computing Technologies, Ramanathapuram, 2014, pp. 673-677.

[7] Theodore S.Rappaport, "Wireless Communication Principles and Practice", Pearson Press, Second edition, 2010.

[8] Proakis. John G., "Digital Communications", McGrawHill, 4th edition, 2001.

[9] S.Coleri, M.Ergen, A.Puri and A.Bahai, "Channel estimation techniques based on pilot arrangemtn in OFDM systems", IEEE Transaction on Broadcasing, vol.48, no.3m pp.223-229, 2002.

[10] Ye(Geoffrey) Li, "Pilot-symbol-aided channel estimation for OFDM in wireless systems", IEEE Transaction on Vehicular Technology, Vol.49, no.4, pp.1207-1215, 2000 .

[11] J.LiandM.Kavehrad, "Effects of time selective multipath fading on OFDM systems for broadband mobile applications," IEEE Communication. Letter., vol. 3, no. 12, pp. 332-334, 1999.

[12] MarvinK.Simon, Mohamed-Slim Alouini, "Digital Communication over Fading Channels", A Unified Approach to Performance Analysis", John Wiley \& Sons, Inc.,2012, Print ISBN :0-471-31779-9, Electronic ISBN: $0 \neg 471-20069-7$.

[13] Digham, F.F., Hasna, M.O., "Performance of OFDM with M-QAM modulation and optimal loading over Rayleigh fading channels", IEEE Conference on Vehicular Technology, Vol.1, pp.479-483 2004, DOI:10.11.09/VETECF-2004.

[14] Xiaoyi Tang, Mohamed-Slim Alouini and Andrea J.Goldsmith, "Effect of Channel Estimation Error on MQAM BER Performance in Rayleigh Fading", IEEE Transaction on Communications, Vol.47, n0.12, 1999.

[15] H. S. Abdel-Ghaffar and S. Pasupathy, "Asymptotical performance of M-ary and binary signals over multipath/multichannel Rayleigh and Rician fading," in IEEE Transactions on Communications, vol. 43, no. 11, pp. 2721-2731, Nov 1995. 